\title{
The role of poliphenols in formation of fungicidal activity and resistance to exometabolites of the fungus on the example of in vitro crops sunflower and savory
}

\author{
Elena Kalashnikova ${ }^{1}$, Svetlana Zaytseva ${ }^{1 *}$, Nguen Tan Thay $^{2}$, Rima Kirakosyan ${ }^{1}$, Mikhail \\ Cherednichenko $^{1}$, Dariya Khlebnikova ${ }^{1}$, and Oksana Polivanova ${ }^{1}$ \\ ${ }^{1}$ Department of biotechnology, Russian State Agrarian University - Moscow Timiryazev Agricultural \\ Academy, Moscow, 127550, Russian Federation \\ ${ }^{2}$ Department biotechnology, Republic of Vietnam, Hanoi, TrauQuy - Gia Lam, Vietnam National \\ University of Agriculture
}

\begin{abstract}
When studying the formation of secondary metabolites represented by phenolic compounds in callus cultures of sunflower plants, it was found that under in vitro conditions under the influence of a stress factor, the accumulation of polyphenols increases. It was found that the biosynthetic activity of callus cultures with respect to polyphenol increases in proportion to the concentration of the culture filtrate of the fungus (Sclerotinia Sclerotiorum) in the nutrient medium. This is confirmed by the qualitative and quantitative determination of the content of polyphenols in callus cultures. In savory plants, when introduced into culture in vitro, there is a sharp decrease in the biosynthesis of phenolic compounds. Ethanol extracts of microclones and callus cultures showed no fungicidal activity.
\end{abstract}

Keywords: poliphenols, fungicidal activity, in vitro, exometabolites.

\section{Introduction}

Due to the peculiarities of the metabolism of the plant organism, where in addition to the reactions of primary metabolism, a variety of substances of secondary origin are synthesized, the extensive therapeutic effect of plant extracts is manifested. The most common secondary compounds are phenolic compounds (bioflavonoids) [1]. The widespread use of polyphenols in pharmacology as biologically active substances is based on their ability to oxidize to form quinine forms, which determines their hepatoprotective, neuroregulatory, capillary strengthening, choleretic and antitumor properties and other properties [2].

As a rule, the most valuable plants for phytopharmacognosia are characterized by a limited range and specific growing conditions and are classified as a small number of endangered species. In this regard, one of the promising areas of biotechnology is the preservation of the biodiversity of rare, medicinal, and endangered plant forms and the

\section{*Corresponding author: smzaytseva@yandex.ru}


creation of in vitro genetic culture banks based on them. In addition, plant cultures in virto are used in biotechnology not only as a model object, but also as a source of valuable biologically active substances that are successfully used in the pharmaceutical industry, as well as for the study of secondary metabolism [3].

Sunflower is traditionally considered the leading oilseed and forage crop, and the share of its cultivation in some regions occupies more than $30 \%$ of all sown areas, maintaining the status of one of the most profitable agricultural crops. In addition, it is widely known about the healing properties of sunflower plants: it describes the use in veterinary medicine of refined refined sunflower oil, as a softening and laxative agent for blockage of the esophagus and goiter in birds, for chronic diseases of the liver and biliary tract, it is used as a basis for the preparation of various dosage forms. In veterinary practice, extracts of sunflower leaves and flowers containing not only plant pigments, but also flavonic glycosides, anthocyanins, bitterness and organic acids are recommended as an antipyretic and improves appetite and digestion [4].

Despite the fact that in the case of sunflower, the predominant product of metabolism is oils, it is of interest for us to study the phenolic metabolism of these plants in order to determine the role of polyphenols in the formation of resistance to the action of mycopathogen.

As is known, the biosynthesis and accumulation of secondary compounds is characterized by plasticity and depends not only on the species of the plant, its organ and the stage of ontogenesis, but also on the growing conditions [5].

Due to the fact that there is not much data in the literature on the features of the formation of secondary substances in the cell cultures of sunflower and savory plants, especially in response to the stress factor, the aim of our study was to study the biosynthetic and fungicidal potential of these plants, and to establish resistance to the highly toxic cultural filtrate of the fungus (SCLEROTINIA SCLEROTIORUM) under in vitro conditions.

\section{Methods and materials}

The material for the work was sunflower plants of the VK 580 genotype, represented by hypocotyl segments isolated from 5-day-old seedlings, as well as savory plants. For surface sterilization of the primary explant, the following sterilizing agents were used: sulema $(0.1 \%)$ and sodium hypochloride $(1.0 \%)$. Dry seeds were kept for 4 to 20 minutes in a sterilizer solution, then washed in three portions of sterile distilled water for 5 minutes each.

Nutrient media. To obtain the primary callus, we used an agarized nutrient medium with mineral salts according to the recipe of Murasiga and Skuga with the addition of vitamins, sucrose and phytohormones in various concentrations and ratios. The $\mathrm{pH}$ of the medium was in the range of 5.6-5.8. The effect of NUC $(0.5-3.0 \mathrm{mg} / \mathrm{l})$ was studied as auxins, and kinetin $(0.5-3.0 \mathrm{mg} / \mathrm{l})$ as cytokinins.

Growing conditions. The isolated tissues were grown in a light room, where the temperature was maintained at $22 \pm 1^{\circ} \mathrm{C}$, under white low-pressure fluorescent lamps LB at an illumination of about $5 \mathrm{KLx}$, air humidity of $70 \%$, and a 16 -hour photoperiod.

Microclones of savory plants cultivated on MS medium, aged 28...35 days were divided into segments and transplanted to media with the addition of Epin $(2.5,12.5$, and $25 \mathrm{mcg} / \mathrm{l}$ of 24-epibrassinolide), MS with the addition of BAP (3 and $5 \mathrm{mg} / \mathrm{l}$ ). Every 14 days, the height of the plants, the number of true leaves, nodes, lateral shoots, the efficiency of root formation were measured, and the morphological features of the plants were noted.

To induce biosynthetic activity, 5-35\% culture filtrate (CF) of the fungus SCLEROTINIA SCLEROTIORUM was added to the nutrient medium. Cultivation of the 
culture of the fungus Sclerotinia sclerotiorum was carried out on an agarized and liquid nutrient medium containing mineral salts according to the $1 / 2$ recipe of Murasig and Skug with the addition of vitamins, sucrose $2 \%$. Preparation of cultural filtrate of the pathogen of the fungus Sclerotinia sclerotiorum. The culture filtrate $(\mathrm{CF})$ of the pathogen was obtained by growing the mushroom isolate in $300 \mathrm{ml}$ flasks in $200 \mathrm{ml}$ volume of liquid culture medium on a rocker with a rotation speed of $100 \mathrm{rpm}$. 108 conidia of the fungus were added to each flask. The culture filtrate was obtained by filtering the mushroom suspension through filter paper and then autoclaving it. The phytotoxicity of $\mathrm{CF}$ and its activity were tested on various sunflower explants: seeds (the length of the root system and the aboveground part of the seedlings were estimated), hypocotyl segments isolated from 5day-old seedlings, as well as on callus culture. To determine the phytotoxicity of the CF pathogen Sclerotinia sclerotiorum, the following concentrations were used: for segments of hypocotyls and callus tissue $-5 \%, 15 \%, 25 \%, 35 \%$ from the final volume of the nutrient medium.

The phytotoxicity of CF was determined by the method of Berestetsky [6] and calculated by the formula 1 :

$$
T=100 \%-\frac{100 l_{o n}}{l_{k}}
$$

(T) - toxicity of CF; (lon)-the sum of the lengths of sunflower seedlings on CF; (lk)-the sum of the lengths of seedlings on water (control).

The fungicidal activity of ethanol extract of callus tissue and savory regenerant plants was determined. Morphogenic callus of light green color was obtained on explants from hypocotyls and cultured on MS medium with the addition of $1.5 \mathrm{mg} / \mathrm{l}$ of BAP for 2 months. Regenerating plants were formed from callus tissue during its cultivation. To prepare the extract, a sample of plant material was ground in a mortar, filled with $10 \mathrm{ml}$ of ethanol and left for 1 hour for extraction. After filtration, the solution was evaporated. The dried extract was diluted with $1 \mathrm{ml}$ of dimethylsulfoxide (DMSO) and added to potato-glucose agar (KGA) - a nutrient medium for the cultivation of phytopathogenic fungi - Fusarium oxysporum TLCA-4. On the 3rd, 5th, and 7th day, the mycelium diameter was measured. As a positive control, the fungicidal mordant "Maxim" (D. V. fludioxonil) was used at a concentration of $83.3 \mathrm{mg} \mathrm{D}$. V./1 of the medium.

To extract the phenolic compounds, the crushed plant material was extracted with hot $96 \%$ ethanol. The content of the sum of soluble phenolic compounds in the extracts was determined by the spectrophotometric method. Calibration curves were constructed according to the routine [7].

The graphs show the arithmetic averages.

\section{Result and discussion}

For many pathogens for which toxins in their pure form have not been isolated or insufficiently studied, the use of culture filtrates (CF) of pathogens is a promising direction for in vitro cell selection for resistance to them. A necessary condition for such an application is the presence of its activity as a selective factor. The following factors are important: method of preparation of CF; aggressiveness of strains introduced into culture; nutrient medium as a factor of accumulation of unknown toxins and preservation of infectious properties of pathogens in culture [8].

Our studies have shown that CF of the fungus Sclerotinia sclerotiorum at different concentrations has a different degree of toxic effect on the germination of sunflower seeds of the studied genotype. When using non-diluted CF $(100 \%)$, its toxicity was more than $50 \%$. A directly proportional correlation was established, which was manifested in the 
following: with an increase in the concentration of CF and the time of cultivation of the pathogen in the suspension culture, the toxicity of the obtained CF increased.

A.

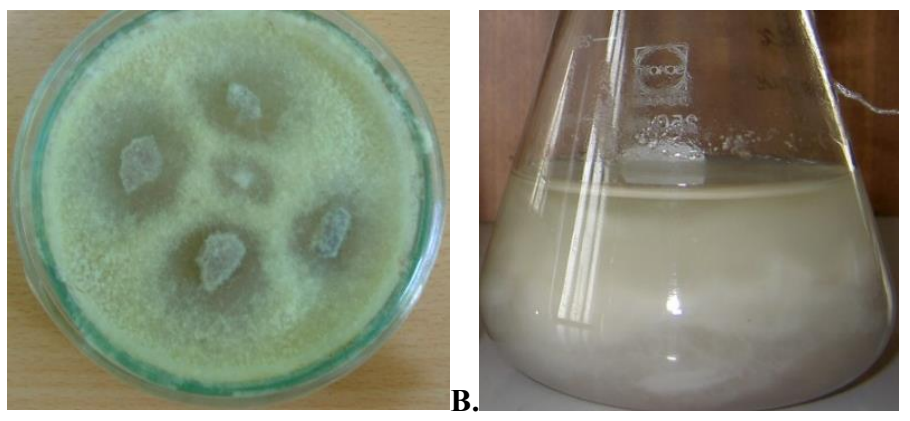

Fig. 1. A. Growth of pure culture of the fungus Sclerotinia sclerotiorum on agarized culture medium and $\mathbf{B}$ cultivation of the fungus Sclerotinia sclerotiorum in liquid culture medium

Under in vitro conditions, plant cells and tissues retain the ability to synthesize various compounds of a secondary nature [9]. To a certain extent, the features of the formation of polyphenols characteristic of the original plants, as well as explants, are also preserved. Phenolic compounds with high biological activity directly affect the processes of formation and growth of the callus mass, including performing a number of protective functions, in particular, in pathogenesis [10].

Earlier, we and other researchers showed that the biosynthetic activity in relation to polyphenols in different organs has significant differences [11]. As a rule, the greatest accumulation of polyphenols in herbaceous actively vegetating plants was observed in the leaves, which is most likely due to the higher biosynthetic ability to form such representatives of phenolic compounds as flavanols in them. The high content of polyphenols in aboveground plant organs is due to the presence of chloroplasts, which are the main site of flavanol synthesis [12]. The same pattern was maintained in the early stages of ontogenesis - in seedlings. The lowest content of polyphenols in sunflower seedlings was observed in the hypocotyl. Under in vitro conditions, the formation of bioflavonoids preserves the organ-specificity characteristic of intact plants, but to a less pronounced degree [13]. In order to minimize the physiological and genotypic features of the accumulation of polyphenols by callus tissues obtained "by inheritance" from the original explants, we selected a callus initiated exclusively from hypocotyl as the object of research.

A.
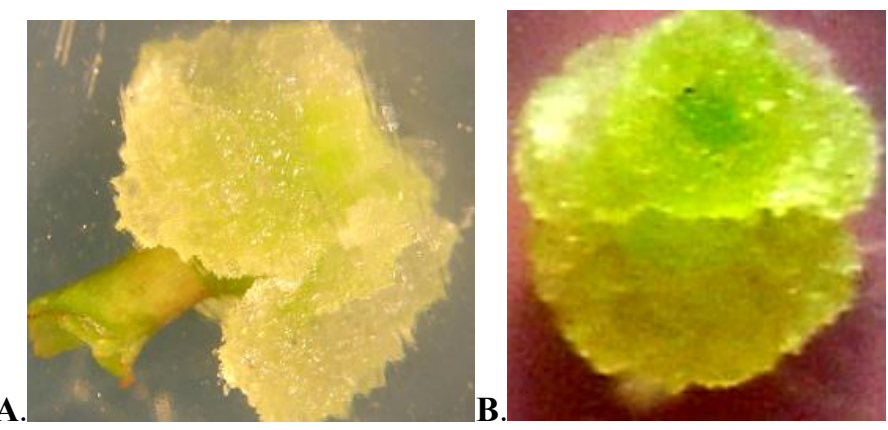

Fig. 2. A. Initiation of callus tissue on the sunflower hypocotyl segment and B. sunflower callus.

In the first series of experiments, we conducted studies to study changes in the quantitative content of phenolic compounds in the primary explant-hypocotyl segments, when introduced into culture in vitro. 
As the callus tissue is cultured under standard in vitro conditions, the total content of soluble FS in sunflower already by the 1st passage sharply decreases in comparison with the primary explant (almost 4 times). So, by the second passage, a slight decrease in the formation of polyphenols continued, and by the third passage, a slight increase was observed. Further culturing of callus tissue under standard conditions did not significantly change the level of phenolic compounds and was in the range of $3.5-5 \mathrm{mg} / \mathrm{g}$ of raw mass, which was only $30 \%$ of the initial level in explants (Fig. 3). A decrease in the biosynthetic activity of callus cultures with respect to polyphenols has been repeatedly noted by other researchers [14]. In most cases, the level of their accumulation is significantly lower than in the original tissues, which may be a consequence of changes in the cytogenetic and biochemical characteristics of cells under in vitro conditions.

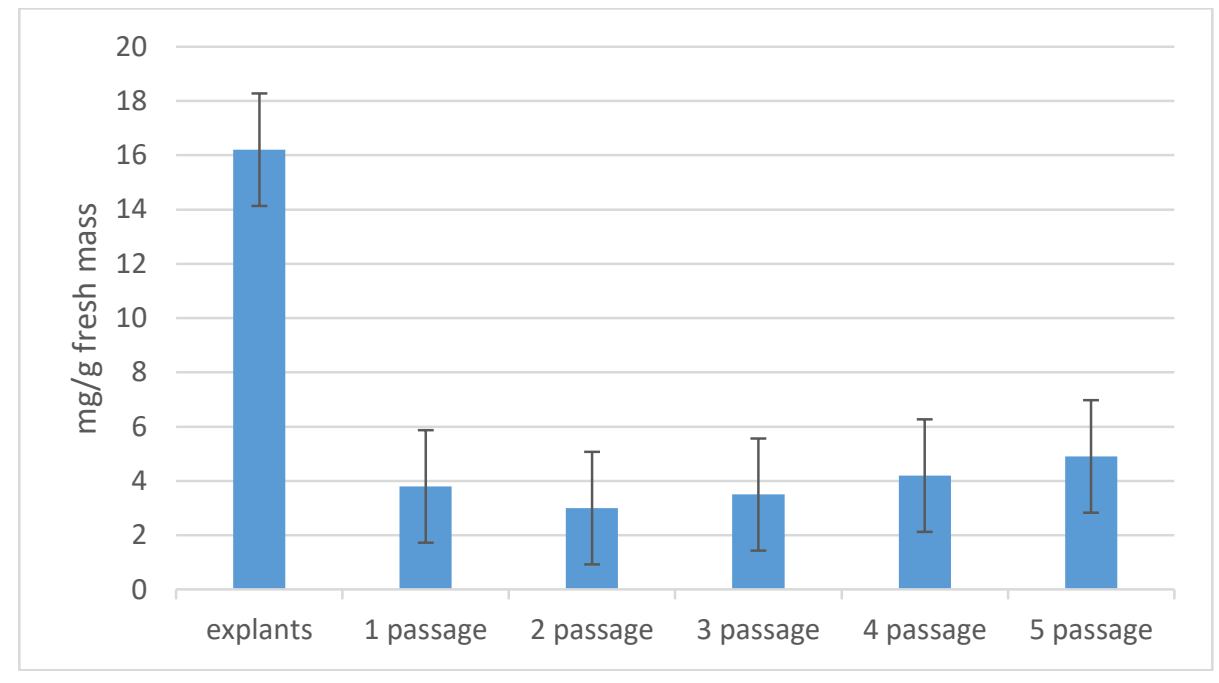

Fig. 3. Changes in the total content of soluble phenolic compounds in explant and callus cultures of sunflower genotype VK 580 during cultivation.

It is known that the biosynthesis of polyphenols in the presence of a stress factor changes [15]. We also noted that when culturing callus tissue under stressful conditions (the presence of $\mathrm{CF}$ of the pathogen in different concentrations), a change in the considered indicator was observed, which was manifested in an increase in the biosynthetic activity of the callus tissue (Fig. 4). Probably, the cultivation of sunflower callus tissue under stressful conditions leads to the "launch" of an increased programmed protective reaction, manifested in an increase in the synthesis of phenolic compounds, as it has been repeatedly noted in the literature [16] The presence of pathogen culture filtrate in increased concentrations ( 25 and $35 \%$ ), led to a change in the quantitative content of soluble phenolic compounds in callus tissue to a greater extent than other concentrations of CF (Fig. 4). For the sunflower genotype VK 580, these changes were proportional to the concentration of the stress factor. As follows from the analysis of literature sources, in plants with pronounced fungicidal activity, substances such as polyphenols with biological activity prevail in the complex of secondary metabolites [17]. 


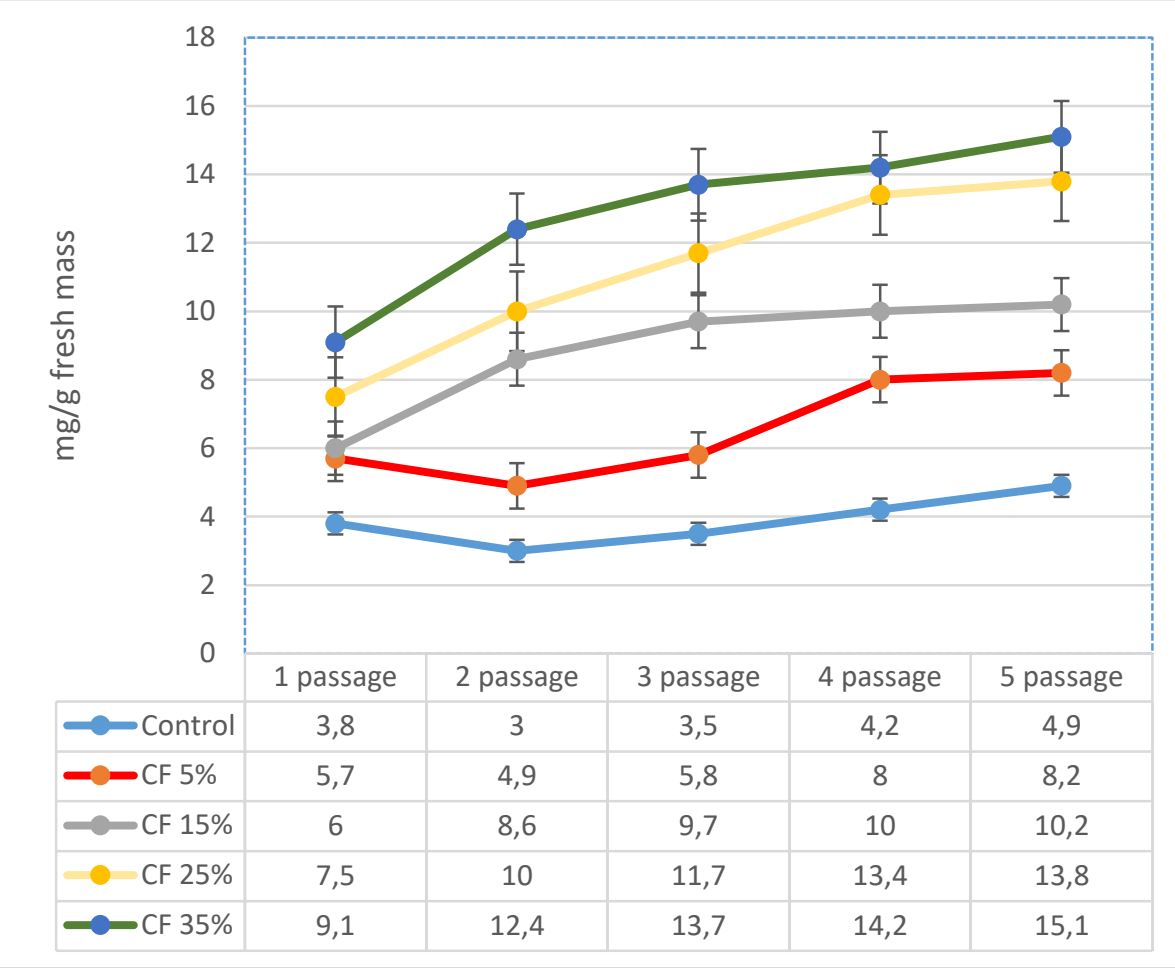

Fig.4. Changes in the total content of soluble phenolic compounds in sunflower callus tissues of the VK 580 genotype during long-term cultivation in the presence of culture filtrate (CF) of the fungus Sclerotinia sclerotiorum of different concentrations.

Therefore, the study of the effect of plant extracts on pathogen toxins is an urgent direction and can serve as an indirect sign of the biosynthetic ability of plants to form secondary substances that have not only biological activity, but also fungicidal action.

For a more detailed study of the processes of changes in the biosynthesis of soluble phenolic compounds occurring in cells of long-term cultured callus cultures on selective media and in the control version, it was important to study the qualitative composition of the phenolic complex during in vitro cultivation. These studies were carried out in the callus tissue of the studied sunflower genotype at the I, III and V passages of the cell selection cycle. As a selective factor, we selected the variant of the presence of the pathogen culture filtrate in the medium at a concentration of $15 \%$. This concentration was chosen not only because of the active growth of callus tissue under these conditions, but also because of a significant increase in the biosynthesis of polyphenols of the studied genotype in response to a stress factor. The results obtained are shown in Figures 5.

As follows from the data shown in the figures, the composition of the phenolic complex changes during the cultivation of the callus tissue of the experimental and control variants.

In the control variant, at the stages of introduction into the culture in vitro (I passage), some depletion in the phenolic spectrum is observed. As the callus tissue was passivated and adapted to the cultivation conditions, we observed an increase in the diversity of substances of a phenolic nature. Moreover, the VK 580 genotype was characterized by the formation of 5 new substances of a flavonol nature. It should be noted that the increase in the phenolic complex was already noted in the first passage. 


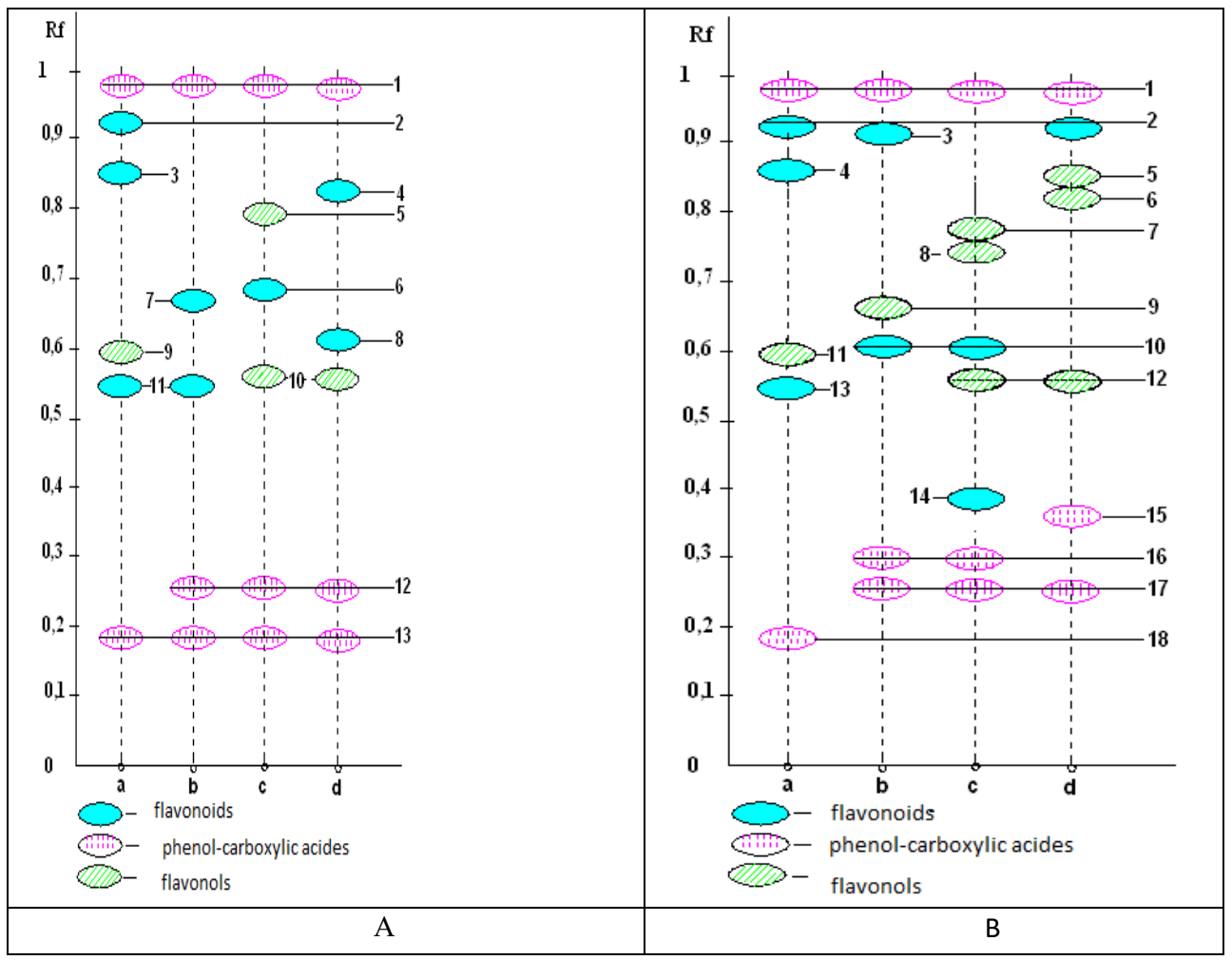

Fig. 5. Chromatogram diagram of ethanol extracts of phenolic compounds of various sunflower tissues of the VK 580 genotype: A-control, B-presence of the CF pathogen Sclerotinia sclerotiorum in the nutrient medium at a concentration of $15 \%$ (a-hypocotyl, b - I passage, c-III passage, $d-\mathrm{V}$ passage)

When culturing callus tissue under stressful conditions, we showed a change in the diversity of the phenolic complex (Fig.5B). In all the studied genotypes, an enrichment of the spectrum of synthesized substances of phenolic nature was observed due to the de novo biosynthesis of phenylpropanoid and flavonoid compounds. Thus, for the VK 580 genotype, by the third passage, the presence of 8 phenolic compounds was noted, compared to 6 in the control sample. It can be assumed that such changes in the qualitative composition are also associated with the quantitative content of polyphenols (Fig. 4). All this indicates that the cultivation of callus tissue under stressful conditions significantly changes the synthesis of phenolic compounds, which leads to the appearance of a more diverse phenolic complex.

In order to support the above data on the role of bioflavonoids in providing the fungicidal properties of plant extracts, we continued experiments with savory plants, which are widely used in pharmacognosy, as fungicides due to their high biosynthetic ability to accumulate polyphenols. Thymol and carvacrol are the main components of the phenolic fraction of essential oils.

The primary task was to elucidate the features of the accumulation of phenolic compounds in in vitro cultures represented by microclones and callus tissues of Satureja hortensis L.. (Fig. 6) The ability to synthesize secondary compounds in the microclones of the studied plants was lower than that of the original tissues. Intact savory plants during the active growing season were characterized by a high accumulation of flavanoids exceeding $49 \mathrm{mg} / \mathrm{g}$ of fresh mass, while microclones accumulated no more than $8.4 \mathrm{mg} / \mathrm{g}$ of fresh mass. At the same time, the biosynthetic organospecificity characteristic of the original 
plant was observed. This once again confirms the data indicating that in vitro conditions maintain an identical tendency to the synthesis of secondary compounds characteristic of intact tissues [9]. It should be noted that the synthesis and accumulation of flavonoids in callus lines is much less intense than in microclones. During 5 passages, there were no statistically significant deviations in the level of flavonoid accumulation in the callus lines from the primary callus. Savory callus cultures accumulated flavonoids in an extremely small amount, not exceeding $0.7 \mathrm{mg} / \mathrm{g}$ of raw weight (Fig. 7). The issue of weakening or complete loss of biosynthetic ability in cell cultures in relation to secondary metabolites, in plants with potentially high biological activity, has been repeatedly discussed in the scientific literature.

A.

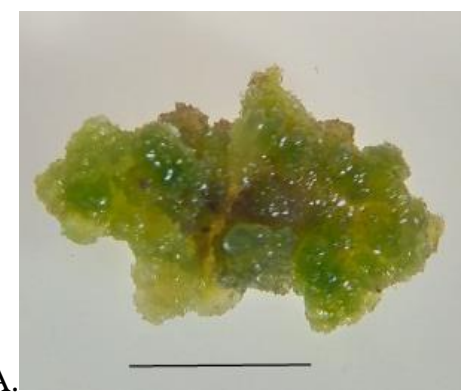

B.

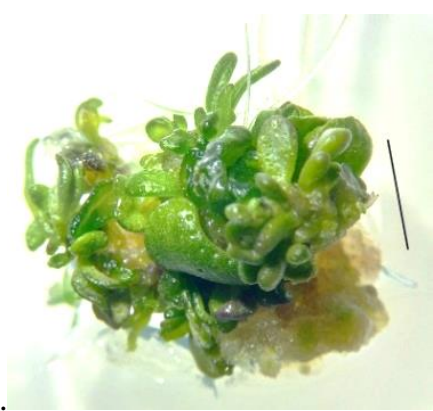

Fig. 6. A - Appearance of the savory callus, B- initiation of morphogenesis on the segment of the savory callus tissue.

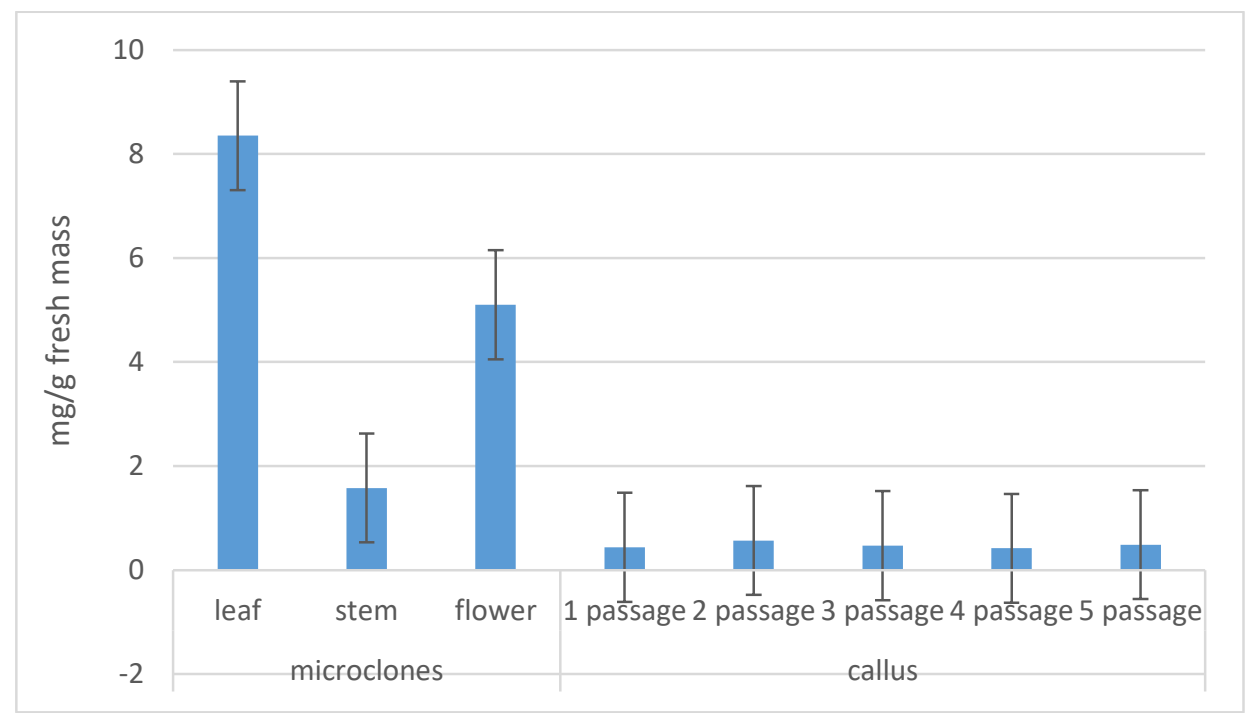

Fig. 7 Changes in the total content of flavonoids in the microculons and callus cultures of savory as they are cultivated.

We studied the effect of ethanol extract from regenerating plants and S. Hortensis callus with an extremely low content of polyphnols on the growth of colonies of phytopathogenic fungi-Fusarium oxysporum TLC-4 (Fig. 8). The data obtained indicate that the addition of plant extract from callus and regenerating plants in all the studied concentrations does not have a fungicidal or fungistatic effect against Fusarium oxysporum TLC-4 fungi. The addition of an extract from regenerating plants at a concentration of $75 \mathrm{mg} / 1$ led to a slight decrease in the diameter of the mycelium of the fungus, and the addition of an extract from 
callus $(75 \mathrm{mg} / \mathrm{l})$ from regenerating plants $(150 \mathrm{mg} / \mathrm{l})$ slightly slowed the growth of Fusarium oxysporum TLC-4.

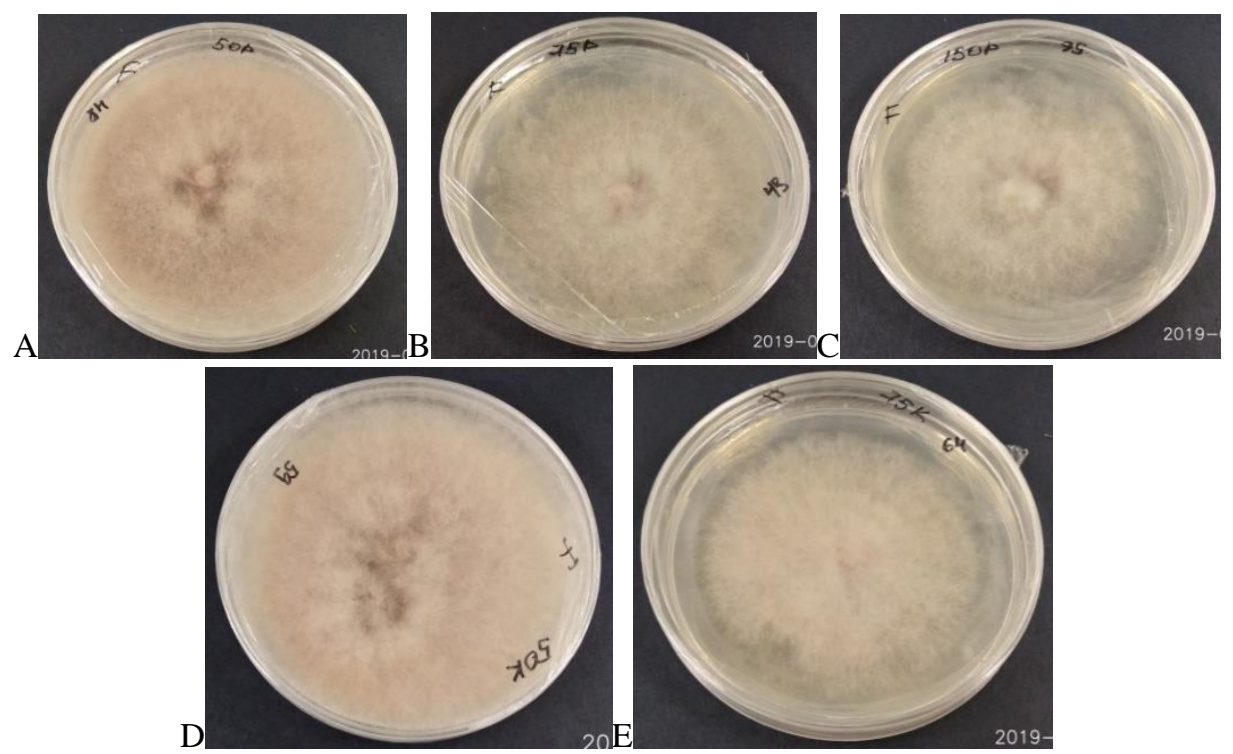

Fig. 8. Colony of the fungus Fusarium oxysporum TLCA-4 on the 7th day of cultivation on a KGA culture medium with the addition of: A-50 mg/l extract from regenerating plants; B-75 mg / 1 of regenerating plant extract; C-150 mg / 1 of regenerating plant extract; D- $50 \mathrm{mg} / 1$ of callus extract; E$75 \mathrm{mg} / 1$ of callus extract

\section{Conclusions}

Based on the above, it can be concluded that callus cultures initiated from plant tissues with minimal ability to biosynthesize phenolic compounds, in response to the stress factor represented by a highly aggressive strain of the fungus Sclerotinia sclerotiorum, show an increase in biosynthetic ability correlated with the concentration of the cultural filtrate of the fungus. It follows from the obtained results that the presence of the cultural filtrate of the pathogen in the medium leads to a change in the composition of phenolic compounds in the direction of its increase in comparison with the control variant, which is confirmed by biochemical studies of the quantitative and qualitative content of polyphenols. Therefore, the study of the physiological and biochemical status of cultures belonging to different taxonomic groups is the most important and primary task.

Undoubtedly, all this is of great practical importance, indicating the role of polyphenols as a protective factor and in the formation of the fungicidal activity of plant extracts used as potential sources of valuable biologically active substances for the pharmaceutical industry. Our studies have shown that secondary metabolites not only contribute to overcoming the inhibitory effect of pathogens on the growth and development of cullus cultures, but also can be stimulators of various morphophysiological processes, as evidenced by a number of literature sources [18].

\section{References}

1. G. M. Alekseeva, Pharmacognosy. Medicinal raw materials of plant and animal origin (SpecLit. Saint-Petersburg. 2013) 
2. V. A. Kurkin, Phenylpropanoids-promising natural biologically active compounds ( State Medical Publishing House. Un-t, Samara, 1996)

3. O. V. Zhurba, M. Ya.. Dmitriev, Medicinal, poisonous and harmful plants ( Kolos Publishing House, Moscow, 2002)

4. Nosov, A.M. Biology of cultured cells and plant biotechnology. Edited by R. G. Butenko (Nauka, Moscow, 1991)

5. E. A. Kalashnikova, S. M.Zaitseva, Doan Thu Thui, R. N.Kirakosyan, Questions of Biological, Medical and Pharmaceutical Chemistry, 1 (2020) Doi: https://doi.org/10.29296/25877313-2020-01-07 1982)

6. O. A. Berestetsky, Methods of experimental mycology (Naukova dumka, Kiev,

7. M. N. Zaprometov, Biochemical methods in plant physiology (Nauka, Moscow,1971)

8. N. M. Araslanova, M. V. Ivebor, S. L. Saukova, T. S. Antonova. Scientific and Technical Bulletin of the All-Russian Research Institute of Oilseeds, 1 (2014)

9. E. A. Kalashnikova, S. M. Zaitseva, Doan Thu Thui, R.N. Kirakosyan, Questions of biological, Medical and Pharmaceutical chemistry, 22, 5 (2019)

10. M. N. Zaprometov Phenolic compounds and their role in plant life. LVI Timiryazev readings (Nauka. Moscow, 1996)

11. Doan Thu Thui, E. A. Kalashnikova, S. M. Zaitseva, R. N. Kirakosyan, Natural and technical Sciences, 2 (2018)

12. M. N. Zametov, T. N. Nikolaeva The ability of isolated chloroplasts from bean leaves to carry out biosynthesis of phenolic compounds, 50, 5 (2003)

13. E. A. Kalashnikova, S. M. Zaitseva, Doan Thu Thui, R. N. Kirakosyan International Scientific Research Journal,Biology, -6 (96), 2 (2020)

14. G. A. Dubravina, Plant Physiology, 52 (2005)

15. M. N. Zaprometov, Phenolic compounds and their role in plant life. LVI Timiryazev readings (Nauka. Moscow, 1996).

16. N. V. Zagoskina, E. A.Goncharuk, A. K. Alyavina, Russian Journal of Plant Physiology. 54 (2007)

17. A. A. Maryin, N.E. Kolomiets, Fundamental and clinical medicine, 2, 4 (2017)

18. E.A. Kalashnikova Cellular engineering of plants: A textbook (YURAYT Publishing House, Moscow, 2020) 